\title{
A STUDY COMPARING THE EFFICACY OF MONOPOLAR RADIOFREQUENCY AND GLYCOLIC ACID PEELS IN FACIAL REJUVENATION OF AGING SKIN USING HISTOPATHOLOGY AND ULTRABIOMICROSCOPIC SONOGRAPHY (UBM) - AN EVIDENCE BASED STUDY
}

\author{
Deepal VWakade', Chitra S Nayak', Kalpana D Bhatt
}

Topiwala National Medical College \& BYL Nair Charitable Hospital, Dr. A. L. Nair Road, Mumbai, Maharashtra, India ${ }^{1,2}$; UBM Institute, Ganesh Baug, Behind Ruia College, Dadar, Mumbai, Maharashtra, India ${ }^{3}$

\begin{abstract}
Summary: Background: Radio frequency (RF) and chemical peels have been used for nonablative skin rejuvenation. Both of these cause collagen remodeling in the dermis and neo-collagen formation resulting in facial rejuvenation. There is limited literature on the evaluation of collagen remodeling by objective methods. Objective: To compare the benefits of monopolar radiofrequency and glycolic acid peels in facial rejuvenation with regards to histopathology and Ultrabiomicroscopic sonography (UBM). Methodology: In this study, forty patients with mild to moderate photoaging received four treatments with 3 weeks interval of monopolar radiofrequency on one side of face and glycolic acid peels in increasing concentrations $\left(\mathrm{Neostrata}^{\mathrm{R}}\right)$ on the other side. Pre and post treatment, $2 \mathrm{~mm}$ biopsies were taken from both preauricular areas and Ultrasonography using a $35 \mathrm{MHz}$ probe was done from outer canthus of eye and nasolabial folds from both sides of face. A blinded assessment was done to measure the increase in the grenz zone and dermal thickness.

Results: In 35/40 patients there was a significant increase in the grenz zone on histopathology and decrease in subepidermal low-echogenic band (SLEB) on UBM of the nasolabial folds on both sides of the face $(\mathrm{p}<0.05)$. Conclusion: Radiofrequency and chemical peels showed equal efficacy in the treatment of facial rejuvenation.
\end{abstract}

Keywords: Radiofrequency; Chemical peels; Grenz zone; SLEB

\section{Introduction}

There are two clinically and biologically distinct aging processes affecting the skin (1). The first is intrinsic aging, which affects the skin by slow, irreversible tissue degeneration. The second is extrinsic aging or photoaging, which is the effect of chronic exposure to ultraviolet radiation on skin. There are a myriad of therapeutic modalities that can improve photoaging. These modalities may be divided into topical agents and procedural agents. Topical agents include retinoids, hydroquinones, and combination therapies. Procedural agents include chemical peels, microdermabrasion, lasers, and intense pulse light and nonablative radiofrequency. Chemical peeling is a cosmetic procedure that involves the application of one or more exfoliating agents to the skin to wound the epidermis and dermis in a controlled manner. Monopolar radiofrequency (RF) is emerging to be a novel treatment in the field of cosmetic dermatology (2). Radiofrequency is the number of oscillations (or waves) per second of the electric and magnetic fields within the radio waves portion of the electromagnetic spectrum. When RF energy is applied to the skin, resistance encountered by the energy flow causes heat to be produced which causes tight- ening to the underlying tissue structures. When comparing RF to other non-ablative (procedures that do not involve the destruction of the outer layer of the skin) techniques, RF energy differs from laser energy in that lasers tends to scatter or absorb into the upper layers of the skin, making it difficult to deliver sufficient heat into the deeper layers without damaging the skin's surface while RF energy is able to penetrate deeper into the skin and affect the deeper dermis and subcutaneous layers causing skin tightening. RF energy produced is not affected by tissue diffraction or absorption by epidermal melanin. As such, RF-based systems are appropriate for any skin type. Both radiofrequency and chemical peels produce subsequent collagen remodeling and skin rejuvenation. The purpose of this study was to assess and objectively quantify the benefits of both the treatments in facial rejuvenation.

\section{Materials and Methods}

Subjects with mild to moderate signs of photoaging in terms of freckles, wrinkles and mild skin laxity in the age group of 35-55 years were enrolled in the study. The study was approved by the institutional review board. Treatment 
and study details were fully explained to subjects, and all signed an informed consent form. Inclusion criteria were bilateral facial changes due to sun damage. Exclusion criteria were photosensitivity to sunlight, any sign of infection or inflammatory skin disease, history of hypertrophic scars or keloids, use of oral isotretinoin or any photosensitizing drugs in the past 6 months. Monopolar radiofrequency was done on the left half of the face and glycolic acid peels in increasing concentrations $(35,50$, and $70 \%)$ were done on the right half at an interval of three weeks. A total of four sittings of each modality were done. Findings were recorded in a clinical proforma.

Device and technique: The radiofrequency machine used in our study was a monopolar radiofrequency, Surgitron Ellman ${ }^{\circledR}$ generating a radiofrequency upto $6 \mathrm{MHz}$, consisting of a hand piece, rejuvenation probe, antenna and foot plate. A ball tip rejuvenation probe of $0.5 \mathrm{~cm}$ by $0.5 \mathrm{~cm}$ was used. A coupling gel as a cooling agent was applied to the skin. When the machine was on, the tip was placed against the skin. Radio frequency current passes from the tip to the return pad and back again. Holding the tip against the skin for the whole treatment cycle which consisted of 10 seconds, caused heating of the skin and tissue beneath it, causing the required contraction and tightening. Therefore in one cycle $(10 \mathrm{sec})$, area of skin measuring $1 \mathrm{~cm}$ by $1 \mathrm{~cm}$ was treated. Each application to an area lasts for 10 seconds and then the probe was moved to the next area. The treatment was continued till mild erythema developed. The entire left half of the face was treated in this manner. Initially a constant frequency of $2.5 \mathrm{MHz}$ was used and then increased according to the patient's tolerability in each visit. A maximum frequency of $4 \mathrm{MHz}$ was used. On the right side of the face, glycolic acid peels $\left(\right.$ Neostrata $\left.^{\mathrm{R}}\right)$ were applied for a total of 4 sittings. The peel cleansing solution (which is generally an alcohol based product) was applied to remove any final debris. Subjects were instructed to keep their eyes closed during the procedure. The peel procedure began by applying the glycolic acid to the face, beginning at the forehead and working it down over the cheeks, chin, nose, and upper cutaneous lip within 20 seconds. If blanching or frosting was encountered in any particular areas, then immediate neutralization was performed at that site. Once the skin achieved a uniform degree of erythema, full face neutralization was done with cool water. In each sitting increasing concentrations were applied $(35,50,70 \%)$ on the right half of the face. After the procedure a constant sunscreen was given to the patient.

Assessment: During the treatment sessions, subjects were monitored for heat discomfort, edema, and intense erythema. Photographs were taken before and after treatment. Assessments were done on the basis of histopathology, UBM and subjective evaluation.

Histopathology: Punch biopsies $(2 \mathrm{~mm})$ were done at baseline and after the last treatment from the pre-auricular areas on both sides of the face. In photoaged skin, elastotic changes and thin collagen bundles are seen in the upper dermis called the "grenz zone" $(3,4)$ (Figure 1). After treat- ment, neo-collagen is deposited in the upper dermis which causes widening of the grenz zone. In our study, the thickest portion of the grenz zone was measured. This parameter was measured using an eye-piece micrometer. An eyepiece micrometer has a series of numbered lines inside of it which make it look like a ruler (x). After placing the special eyepiece, a calibration slide is used which is a glass slide $0.01 \mathrm{~mm}$ engraved on to its top surface(y). The eyepiece and the slide are mounted on the microscope on $40 \times$ and the number of lines are counted. The distance between each line of the eyepiece is calculated using an equation: $y / x \times 10$ $=$ measurement between two lines.

Ultrabiomicroscopic sonography (UBM): A $35 \mathrm{mHz}$ ultrasonography probe of Paradigm ${ }^{\circledR}$ was placed against the outer canthus of eye and nasolabial folds from both sides of

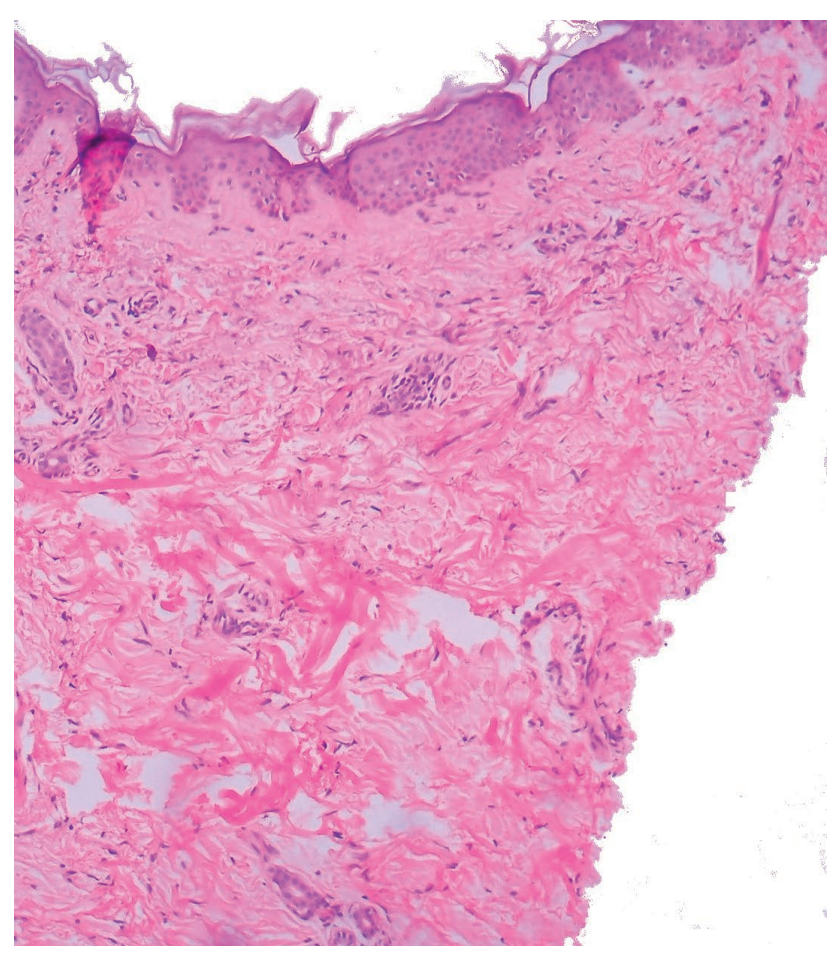

Fig. 1: 40× H\&E: Histology of aging skin showing grenz zone.

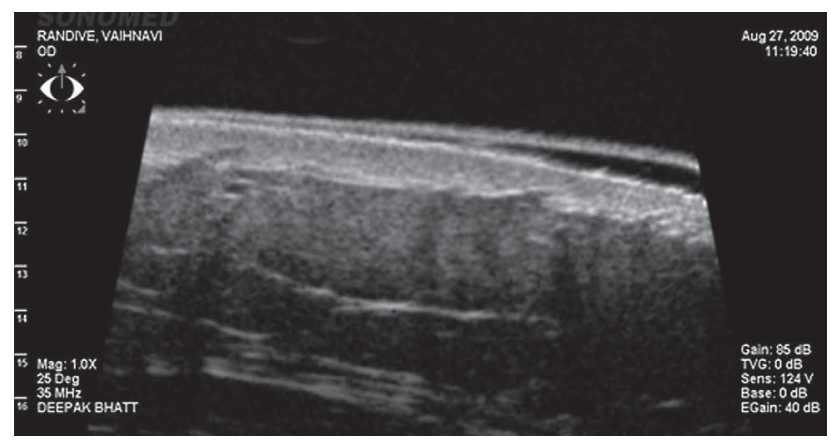

Fig. 2: Normal sonography skin showing epidermal entrance echo and dermal thickness (hyperechoeic region). 
face to assess SLEB. The SLEB (subepidermal low-echogenic band) is defined as a clearly visual low echogenic band in the upper dermis immediately below the epidermal entrance echo. It is usually present in aging skin and is due to the degenerative changes in upper dermis. SLEB is seen in aging skin in $50 \%$ of individuals (3). After treatment the SLEB decreases and may disappear also because of collagen deposition $(5,6)$. The SLEB was measured with calipers inbuilt in the software. The images were captured on the software (Figure 2).

Subjective evaluation: All patients were asked to grade their clinical response after the treatment according to the treatment response scale $(0=$ no improvement, $1=1-25 \%$ improvement, $2=26-50 \%$ improvement, $3=51-75 \%$ improvement, and $4=76-100 \%$ improvement).

Statistical analysis: Quantitative and qualitative measurements were analyzed using the software package for statistical science (SPSS for Windows). Data were analyzed using the Greenhouse-Geisser test and Mann-Whitney test for parametric and non-parametric data respectively. Statistical significance was defined as P less than or equal to 0.05 .

\section{Results}

Clinical evaluation: Out of 40 patients, only 35 patients completed the study. All 35 patients showed some clinical improvement of skin tightening (Figure 3) All subjects were asked to grade the improvement after the last treatment according to the treatment response scale. Only 3 patients had $>50 \%$ improvement on the chemical peel treated side and 24 patients had $>50 \%$ improvement on the radiofrequency treated side. The mean subjective evaluation was more on the radiofrequency treated side than the chemical peel treated side. However the comparison was not statistically significant.

Histopathological evaluation: On microscopic examination of hematoxylin-eosin stained sections various

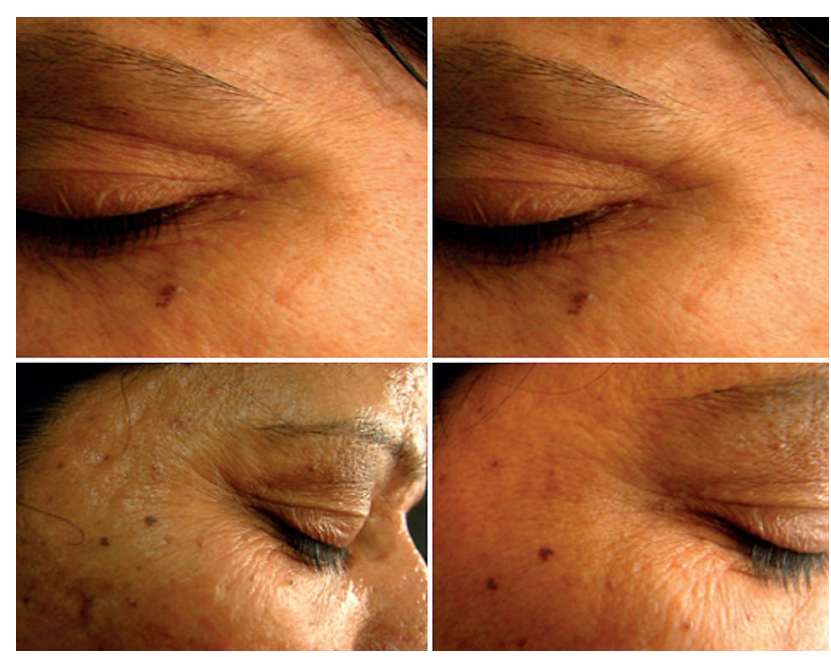

Fig. 3: Pretreatment (left) and posttreatment (right) on radiofrequency and chemical peel treated side. parameters like epidermal thickness and grenz zone were assessed. The mean epidermal thickness on the chemical peel treated side decreased from $41.07 \mu$ to $35.50 \mu$ and the mean epidermal thickness on the radiofrequency side decreased from $43.57 \mu$ to $37.85 \mu$. This decrease within the chemical peel and radiofrequency group was statistically significant by applying the Greenhouse-geisser test of significance. The mean grenz zone increased from $23.75 \mu$ to $57.94 \mu$ after 4 sittings on the chemical peel treated side. The mean grenz zone increased from $25.92 \mu$ to $57.50 \mu$ after 4 sittings on the radiofrequency treated side. This increase within the chemical peel and radiofrequency group was statistically significant. However the comparison of the above parameters between the radiofrequency and chemical peel side was not statistically significant by applying the same test. (Figure 4a,b).

The treatment response on histopathology was assessed by an independent observer also. There was a correlation of more than $98 \%$ between the investigator and blinded observer.

\section{A}
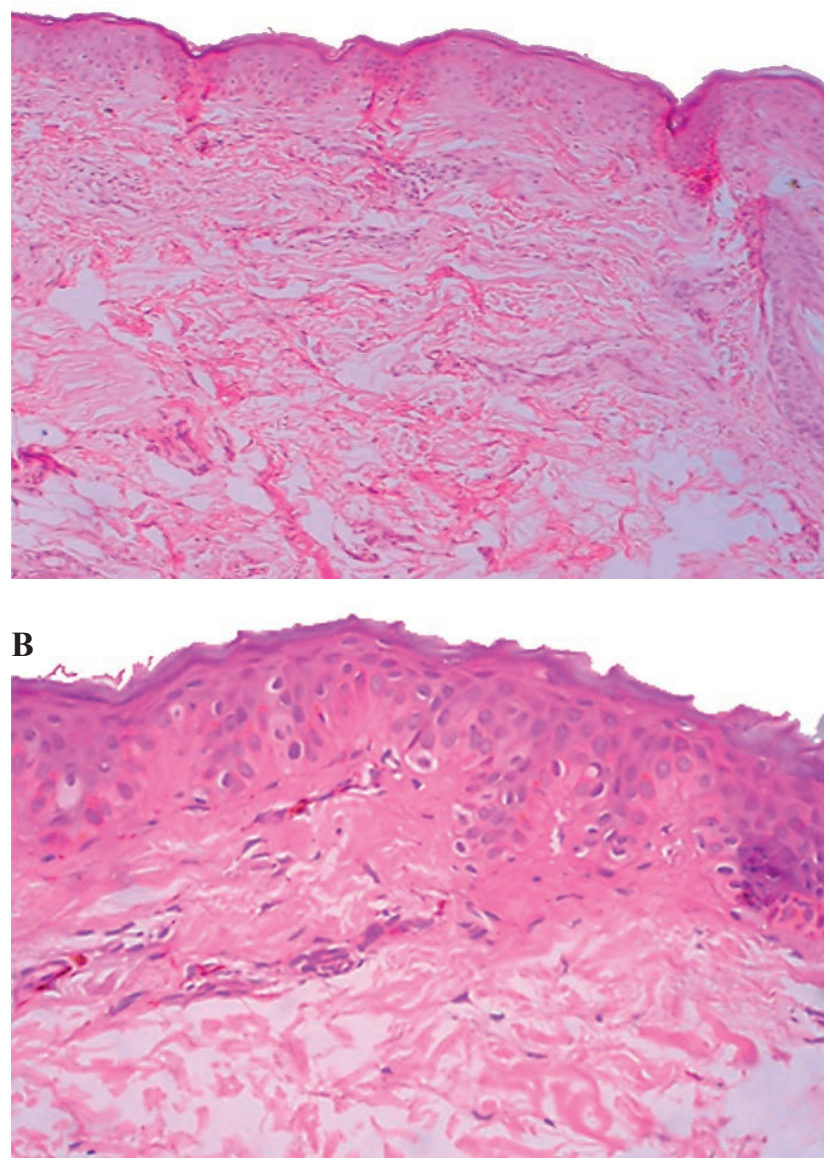

Fig. 4: Increase in the grenz zone after treatment.

(a) $10 \times$ H\&E: Baseline: Histology of aging skin showing epidermal hyperplasia and disorganization of collagen bundles.

(b) 40× H\&E: Box showing a well developed grenz zone seen. 


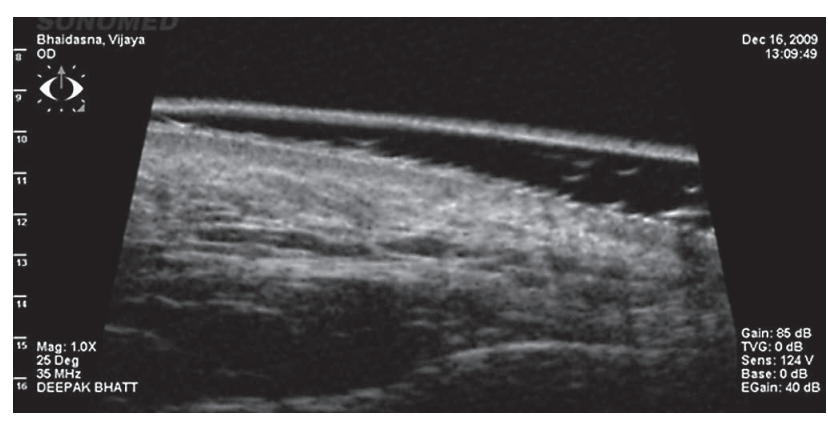

Fig. 5: Sonography shows absence of SLEB.

Ultrasonography evaluation: On UBM, subepidermal low-echogenic band (SLEB) was assessed. The mean SLEB from lateral canthus on the chemical peel treated side decreased $0.34 \mathrm{~mm}$ to $0.31 \mathrm{~mm}$ and decreased from $0.31 \mathrm{~mm}$ to $0.30 \mathrm{~mm}$ on the radiofrequency treated side. The decrease in SLEB pre and post treatment was not statistically significant by applying the Greenhouse-geisser test of significance. The mean SLEB from nasolabial fold from the radiofrequency treated side decreased $0.32 \mathrm{~mm}$ to $0.25 \mathrm{~mm}$ and from $0.30 \mathrm{~mm}$ to $0.21 \mathrm{~mm}$ on the chemical peel treated side. The decrease in SLEB was statistically significant on both sides. However the comparison of the above parameters between the radiofrequency and chemical peel side was not statistically significant by applying the same test (Figure 5).

\section{Discussion}

Facial rejuvenation is a science which focuses on the use of various techniques: ablative or non-ablative to yield impressive results. In the past, ablative techniques were used to achieve the same but due to disadvantages like increased downtime and prolonged recovery, a search for nonablative techniques were done. There is now an increased interest in a wide range of nonablative treatments of skin aging, which are used to rejuvenate skin with minimal downtime and complications. There are numerous studies on nonablative facial rejuvenation in literature documenting treatment response by subjective methods like photography (7).

There are a very few studies where objective parameters have been used to assess collagen remodelling. We used quantitative evaluation on histopathology and UBM at baseline and at the end of treatment to objectively evaluate the efficacy of anti-ageing procedures.

On histological analysis of pre and post procedure biopsies, we documented a significant mean decrease in the epidermal thickness on the chemical peel and radiofrequen- cy treated side. Actually in reality, chronic UVB irradiation produces an irregular hypertrophy of the epidermis and rejuvenation treatment leads to normalisation of the epidermis (8). We postulate that the decrease in the thickness of the epidermis in our study is due to the normalisation of the epidermis and not true atrophy. There was a significant increase in the mean grenz zone on both sides of the face indicating a collagen deposition induced by radiofrequency and glycolic acid peels. There are no studies evaluating treatment response by measuring grenz zone in literature. Although radiofrequency and chemical peels have been used in various studies for facial rejuvenation few have histologically analyzed the skin of the subjects treated.

On sonography, we documented a decrease in SLEB between both the treatment groups on the nasolabial folds and the lateral canthus, however we found a significant decrease only on the nasolabial folds. There is no published study which has measured decrease in SLEB in literature. We found an increase in dermal thickness on the radiofrequency treated side. Ultrasonography assessment studies of radiofrequency and glycolic acid peels for facial rejuvenation have not been reported. There is no published study which has measured decrease in SLEB in literature. However we conclude that SLEB and Grenz zone are significant assessment parameters to study the effects of anti ageing pocedures on ageing skin.

In conclusion, both monopolar radiofrequency and glycolic acid peels are equally efficacious in facial rejuvenation. In our study we have made a sincere effort to quantitate improvement objectively.

\section{References}

1. Gilchrest BA. Skin Aging 2003: Recent advances and current concepts. Cutis 2003; 72(Suppl 3): 5-10.

2. El-Domyati M, el-Ammawi TS, Medhat W, et al. Radiofrequency facial rejuvenation: Evidence-based effect. J Am Acad Dermatol 2011 Mar; 64(3): 524-35.

3. Montagna W, Kirchner S, Carlisle K. Histology of sun-damaged human skin. J Am Acad Dermatol 1989; 21: 907-18.

4. Warren R, Gartstein V, Kligman AM et al. Age, sunlight, and facial skin: A histologic and quantitative study. J Am Acad Dermatol 25: 751-60.

5. Gniadecka M, Jemec GBE. Quantitative evaluation of chronological ageing and photoageing in vivo: studies on skin echogenicity and thickness. Br J Dermatol 1998; 139: 815-21.

6. de Rigal J, Escoffier C, Querleux B, et al. Assessment of Aging of the Human Skin by In Vivo Ultrasonic Imaging. J Invest Dermatol 1989 Nov; 93(5): 621-5.

7. Goldberg DJ, Rogachefsky AS, Silapunt S. Non-ablative laser treatment of facial rhytides: a comparison of 1450 diode laser treatment with dynamic cooling device as opposed to treatment with dynamic cooling alone. Lasers Surg Med 2002;30: 79-81.

8. Isoda M, Ueda S, Imayama S, et al. New formulation of chemical peeling agent: histological evaluation in sun-damaged skin model in hairless mice. J Dermatol Sci. 2001 Aug; 27(Suppl 1): S60-7.

Received: 06/08/2015

Accepted in revised form: 18/12/2015

\section{Corresponding author:}

Chitra S Nayak, Topiwala National Medical College \& BYL Nair Charitable Hospital, Dr. A. L. Nair Road, Mumbai, Maharashtra 400008, India; e-mail: chitra1202@yahoo.co.in 